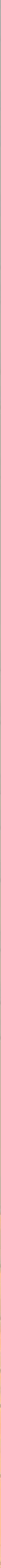




\title{
Lefschetz theorem for abelian fundamental group with modulus
}

\author{
Moritz Kerz and Shuji Saito
}

\begin{abstract}
We prove a Lefschetz hypersurface theorem for abelian fundamental groups allowing wild ramification along some divisor. In fact, we show that isomorphism holds if the degree of the hypersurface is large relative to the ramification along the divisor.
\end{abstract}

\section{Statement of main results}

Lefschetz hyperplane theorems represent an important technique in the study of Grothendieck's fundamental group $\pi_{1}(X)$ of an algebraic variety $X$ (we omit base points for simplicity). Roughly speaking, one gets an isomorphism of the form

$$
\iota_{Y / X}: \pi_{1}(Y) \stackrel{\sim}{\rightarrow} \pi_{1}(X)
$$

for a suitable hypersurface section $Y \rightarrow X$ if $\operatorname{dim}(X) \geq 3$. Purely algebraic Lefschetz theorems for projective varieties satisfying certain regularity assumptions were developed in [SGA 2 1968]. The case of nonproper varieties $X$ and $Y$ is more intricate because one needs a precise control of the ramification at the infinite locus. We show in the present note that for the abelian quotient of the fundamental group a Lefschetz hyperplane theorem does in fact hold. Our basic technical ingredient is the higherdimensional ramification theory of Brylinski, Kato and Matsuda, which is recalled in Section 2. We expect that there is a noncommutative analog of our Lefschetz theorem, which should have applications to $\ell$-adic representations of fundamental groups, especially over finite fields as studied in [Esnault and Kerz 2012].

To formulate our main result, let $X$ be a normal variety over a perfect field $k$, and let $U \subset X$ be an open subset such that $X \backslash U$ is the support of an effective Cartier divisor on $X$. Let $D$ be an effective Cartier divisor on $X$ with support in $X \backslash U$. We introduce the abelian fundamental group $\pi_{1}^{\mathrm{ab}}(X, D)$ as a quotient of $\pi_{1}^{\mathrm{ab}}(U)$ classifying abelian étale coverings of $U$ with ramification bounded by $D$. More precisely, for an integral curve $Z \subset U$, let $Z^{N}$ be the normalization of the closure of $Z$ in $X$ with $\phi_{Z}: Z^{N} \rightarrow X$, the natural map. Let $Z_{\infty} \subset Z^{N}$ be the finite set

Keywords: fundamental group, ramification, Lefschetz theorem . 
of points $x$ such that $\phi_{Z}(x) \notin U$. Then $\pi_{1}^{\mathrm{ab}}(X, D)$ is defined as the Pontryagin dual of the group $\mathrm{fil}_{D} H^{1}(U)$ of continuous characters $\chi: \pi_{1}^{\mathrm{ab}}(U) \rightarrow \mathbb{Q} / \mathbb{Z}$ such that, for any integral curve $Z \subset U$, its restriction $\left.\chi\right|_{Z}: \pi_{1}^{\mathrm{ab}}(Z) \rightarrow \mathbb{Q} / \mathbb{Z}$ satisfies the following inequality of Cartier divisors on $Z^{N}$ :

$$
\sum_{y \in Z_{\infty}} \operatorname{art}_{y}\left(\left.\chi\right|_{Z}\right)[y] \leq \phi_{Z}^{*} D
$$

where $\operatorname{art}_{y}\left(\left.\chi\right|_{Z}\right) \in \mathbb{Z}_{\geq 0}$ is the Artin conductor of $\left.\chi\right|_{Z}$ at $y \in Z_{\infty}$ and $\phi_{Z}^{*} D$ is the pullback of $D$ by the natural map $\phi_{Z}: Z^{N} \rightarrow X$.

Such a global measure of ramification in terms of curves has been first considered by Deligne and Laumon; see [Laumon 1981].

Now assume that $X$ is smooth projective over $k$ (we fix a projective embedding) and that $C=X \backslash U$ is a simple normal crossing divisor. Let $Y$ be a smooth hypersurface section such that $Y \times{ }_{X} C$ is a reduced simple normal crossing divisor on $Y$, and write $\operatorname{deg}(Y)$ for the degree of $Y$ with respect to the fixed projective embedding of $X$. Set $E=Y \times_{X} D$. Then one sees from the definition that the map $Y \cap U \rightarrow U$ induces a natural map

$$
\iota_{Y / X}: \pi_{1}^{\mathrm{ab}}(Y, E) \rightarrow \pi_{1}^{\mathrm{ab}}(X, D) .
$$

Our main theorem says:

Theorem 1.1. Assume that $Y$ is sufficiently ample for $(X, D)$ (see Definition 3.1). If $d:=\operatorname{dim}(X) \geq 3, \iota_{Y / X}$ is an isomorphism. If $d=2, \iota_{Y / X}$ is surjective.

The prime-to- $p$ part of the theorem is due to [Schmidt and Spieß 2000], where $p=\operatorname{ch}(k)$. Below we see that $Y$ is sufficiently ample if $\operatorname{deg}(Y) \gg 0$.

Corollary 1.2. Let $X$ be a normal proper variety over a finite field $k$. Then $\pi_{1}^{\mathrm{ab}}(X, D)^{0}$ is finite, where

$$
\pi_{1}^{\mathrm{ab}}(X, D)^{0}=\operatorname{Ker}\left(\pi_{1}^{\mathrm{ab}}(X, D) \rightarrow \pi_{1}^{\mathrm{ab}}(\operatorname{Spec}(k))\right) .
$$

Proof. In case $X$ and $X \backslash U$ satisfy the assumption of Theorem 1.1, the corollary follows from the corresponding statement for curves. The finiteness in the curves case is a consequence of class field theory. For the general case, one can take by [de Jong 1996] an alteration $f: X^{\prime} \rightarrow X$ such that $X^{\prime}$ and $X^{\prime} \backslash U^{\prime}$ with $U^{\prime}=f^{-1}(U)$ satisfy the assumption of Theorem 1.1. Then the assertion follows from the fact that the map $f_{*}: \pi_{1}^{\mathrm{ab}}\left(U^{\prime}\right) \rightarrow \pi_{1}^{\mathrm{ab}}(U)$ has a finite cokernel.

Corollary 1.2 can also be deduced from [Raskind 1995, Theorem 6.2]. It has recently been generalized to the noncommutative setting by Deligne; see [Esnault and Kerz 2012].

Theorem 1.1 is a central ingredient in our paper [Kerz and Saito 2013]. There we use it to construct a reciprocity isomorphism between a Chow group of zero 
cycles with modulus and the abelian fundamental group with bounded ramification. In fact, Theorem 1.1 allows us to restrict to surfaces in this construction.

\section{Review of ramification theory}

First we review local ramification theory. Let $K$ denote a henselian discrete valuation field of $\operatorname{ch}(K)=p>0$ with the ring $0_{K}$ of integers and residue field $\kappa$. Let $\pi$ be a prime element of $\mathfrak{O}_{K}$ and $\mathfrak{m}_{K}=(\pi) \subset \mathfrak{O}_{K}$ the maximal ideal. By the ArtinSchreier-Witt theory, we have a natural isomorphism for $s \in \mathbb{Z}_{\geq 1}$,

$$
\delta_{s}: W_{s}(K) /(1-F) W_{s}(K) \stackrel{\sim}{\rightarrow} H^{1}\left(K, \mathbb{Z} / p^{s} \mathbb{Z}\right),
$$

where $W_{s}(K)$ is the ring of Witt vectors of length $s$ and $F$ is the Frobenius. We have the Brylinski-Kato filtration indexed by integers $m \geq 0$

$$
\mathrm{fil}_{m}^{\log } W_{s}(K)=\left\{\left(a_{s-1}, \ldots, a_{1}, a_{0}\right) \in W_{s}(K) \mid p^{i} v_{K}\left(a_{i}\right) \geq-m\right\},
$$

where $v_{K}$ is the normalized valuation of $K$. In this paper, we use its nonlog version introduced by Matsuda [1997]:

$$
\mathrm{fil}_{m} W_{s}(K)=\mathrm{fil}_{m-1}^{\log } W_{s}(K)+V^{s-s^{\prime}} \mathrm{fil}_{m}^{\log } W_{s^{\prime}}(K),
$$

where $s^{\prime}=\min \left\{s, \operatorname{ord}_{p}(m)\right\}$. We define ramification filtrations on $H^{1}(K):=$ $H^{1}(K, \mathbb{Q} / \mathbb{Z})$ as

$$
\begin{aligned}
& \mathrm{fil}_{m}^{\log } H^{1}(K)=H^{1}(K)\left\{p^{\prime}\right\} \oplus \bigcup_{s \geq 1} \delta_{s}\left(\mathrm{fil}_{m}^{\log } W_{s}(K)\right) \quad(m \geq 0), \\
& \mathrm{fil}_{m} H^{1}(K)=H^{1}(K)\left\{p^{\prime}\right\} \oplus \bigcup_{s \geq 1} \delta_{s}\left(\mathrm{fil}_{m} W_{s}(K)\right) \quad(m \geq 1),
\end{aligned}
$$

where $H^{1}(K)\left\{p^{\prime}\right\}$ is the prime-to- $p$ part of $H^{1}(K)$. We note that this filtration is shifted by one from the filtration of Matsuda [1997, Definition 3.1.1]. We also let fil $_{0} H^{1}(K)$ be the subgroup of all unramified characters.

Definition 2.1. For $\chi \in H^{1}(K)$, we denote the minimal $m$ with $\chi \in$ fil $_{m} H^{1}(K)$ by $\operatorname{art}_{K}(\chi)$ and call it the Artin conductor of $\chi$.

We have the following facts (cf. [Kato 1989; Matsuda 1997]):

Lemma 2.2. (1) fil $H_{1} H^{1}(K)$ is the subgroup of tamely ramified characters.

(2) $\mathrm{fil}_{m} H^{1}(K) \subset \mathrm{fil}_{m}^{\log } H^{1}(K) \subset \mathrm{fil}_{m+1} H^{1}(K)$.

(3) $\mathrm{fil}_{m} H^{1}(K)=\mathrm{fil}_{m-1}^{\log } H^{1}(K)$ if $(m, p)=1$.

The structure of graded quotients

$$
\operatorname{gr}_{m} H^{1}(K)=\mathrm{fil}_{m} H^{1}(K) / \mathrm{fil}_{m-1} H^{1}(K) \quad(m>1)
$$


is described as follows. Let $\Omega_{K}^{1}$ be the absolute Kähler differential module, and put

$$
\mathrm{fil}_{m} \Omega_{K}^{1}=\mathfrak{m}_{K}^{-m} \otimes_{\mathfrak{O}_{K}} \Omega_{\mathscr{O}_{K}}^{1} .
$$

We have an isomorphism

$$
\operatorname{gr}_{m} \Omega_{K}^{1}=\text { fil }_{m} \Omega_{K}^{1} / \mathrm{fil}_{m-1} \Omega_{K}^{1} \simeq \mathfrak{m}_{K}^{-m} \Omega_{\mathscr{O}_{K}}^{1} \otimes_{\mathfrak{O}_{K}} \kappa .
$$

We have the maps

$$
F^{s} d: W_{s}(K) \rightarrow \Omega_{K}^{1}, \quad\left(a_{s-1}, \ldots, a_{1}, a_{0}\right) \mapsto \sum_{i=0}^{s-1} a_{i}^{p^{i}-1} d a_{i},
$$

and one can check $F^{s} d\left(\right.$ fil $\left._{n} W_{s}(K)\right) \subset$ fil $_{n} \Omega_{K}^{1}$.

Theorem 2.3 [Matsuda 1997]. The maps $F^{s} d$ factor through $\delta_{s}$ and induce a natural map

$$
\mathrm{fil}_{n} H^{1}(K) \rightarrow \mathrm{fil}_{n} \Omega_{K}^{1},
$$

which induces for $m>1$ an injective map (called the refined Artin conductor for $K$ )

$$
\operatorname{art}_{K}: \operatorname{gr}_{n} H^{1}(K) \hookrightarrow \operatorname{gr}_{n} \Omega_{K}^{1} .
$$

Next we review global ramification theory. Let $X$ and $C$ be as in the introduction, and fix a Cartier divisor $D$ with $|D| \subset C$. We recall the definition of $\pi_{1}^{\mathrm{ab}}(X, D)$. We write $H^{1}(U)$ for the étale cohomology group $H^{1}(U, \mathbb{Q} / \mathbb{Z})$, which is identified with the group of continuous characters $\pi_{1}^{\mathrm{ab}}(U) \rightarrow \mathbb{Q} / \mathbb{Z}$.

Definition 2.4. We define $\mathrm{fil}_{D} H^{1}(U)$ to be the subgroup of $\chi \in H^{1}(U)$ satisfying this condition. For all integral curves $Z \subset X$ not contained in $C$, its restriction $\left.\chi\right|_{Z}: \pi_{1}^{\mathrm{ab}}(Z) \rightarrow \mathbb{Q} / \mathbb{Z}$ satisfies the following inequality of Cartier divisors on $Z^{N}$ :

$$
\sum_{y \in Z_{\infty}} \operatorname{art}_{y}\left(\left.\chi\right|_{Z}\right)[y] \leq \phi_{Z}^{*} D
$$

where $\operatorname{art}_{y}\left(\left.\chi\right|_{Z}\right) \in \mathbb{Z}_{\geq 0}$ is the Artin conductor of $\left.\chi\right|_{Z}$ at $y \in Z_{\infty}$ and $\phi_{Z}^{*} D$ is the pullback of $D$ by the natural map $\phi_{Z}: Z^{N} \rightarrow X$. Define

$$
\pi_{1}^{\mathrm{ab}}(X, D)=\operatorname{Hom}\left(\mathrm{fil}_{D} H^{1}(U), \mathbb{Q} / \mathbb{Z}\right),
$$

endowed with the usual profinite topology of the dual.

For the rest of this section, we assume that $X$ is smooth and $C$ is a simple normal crossing. Let $I$ be the set of generic points of $C$, and let $C_{\lambda}=\overline{\{\lambda\}}$ for $\lambda \in I$. Write

$$
D=\sum_{\lambda \in I} m_{\lambda} C_{\lambda}
$$


For $\lambda \in I$, let $K_{\lambda}$ be the henselization of $K=k(X)$ at $\lambda$. Note that $K_{\lambda}$ is a henselian discrete valuation field with residue field $k\left(C_{\lambda}\right)$.

Proposition 2.5. We have

$$
\mathrm{fil}_{D} H^{1}(U)=\operatorname{Ker}\left(H^{1}(U) \rightarrow \bigoplus_{\lambda \in I} H^{1}\left(K_{\lambda}\right) / \operatorname{fil}_{m_{\lambda}} H^{1}\left(K_{\lambda}\right)\right) .
$$

Proof. This is a consequence of ramification theory developed in [Kato 1989; Matsuda 1997]. See [Kerz and Saito 2013, Corollary 2.7] for a proof.

Proposition 2.6. Fix $\lambda \in I$ such that $m_{\lambda}>1$ in (2-5). The refined Artin conductor $\operatorname{art}_{K_{\lambda}}$ (cf. Theorem 2.3) induces a natural injective map

$$
\operatorname{art}_{C_{\lambda}}: \operatorname{fil}_{D} H^{1}(U) / \operatorname{fil}_{D-C_{\lambda}} H^{1}(U) \hookrightarrow H^{0}\left(C_{\lambda}, \Omega_{X}^{1}(D) \otimes \odot_{X} \mathcal{O}_{C_{\lambda}}\right),
$$

which is compatible with pullback along maps $f: X^{\prime} \rightarrow X$ of smooth varieties with the property that $f^{-1}(C)$ is a reduced simple normal crossing divisor.

Proof. This follows from the integrality result [Matsuda 1997, Corollary 4.2.2] of the refined Artin conductor.

Proposition 2.6 motivates us to introduce the following log-variant of $\mathrm{fil}_{D} H^{1}(U)$ :

Definition 2.7. We define $\operatorname{fil}_{D}^{\log } H^{1}(U)$ as

$$
\mathrm{fil}_{D}^{\log } H^{1}(U)=\operatorname{Ker}\left(H^{1}(U) \rightarrow \bigoplus_{\lambda \in I} H^{1}\left(K_{\lambda}\right) / \mathrm{fil}_{m_{\lambda}}^{\log } H^{1}\left(K_{\lambda}\right)\right) .
$$

Lemma 2.8. (1) $\mathrm{fil}_{C} H^{1}(U)$ is the subgroup of tamely ramified characters.

(2) fil $_{D} H^{1}(U) \subset \operatorname{fil}_{D}^{\log } H^{1}(U) \subset$ fil $_{D+C} H^{1}(U)$.

(3) $\mathrm{fil}_{D} H^{1}(U)=\mathrm{fil}_{D-C}^{\log } H^{1}(U)$ if $\left(m_{\lambda}, p\right)=1$ for all $\lambda \in I$.

Proof. This is a direct consequence of Lemma 2.2.

\section{Proof of the main theorem}

Let $X$ be a smooth projective variety over a perfect field of characteristic $p>0$ and $C \subset X$ a reduced simple normal crossing divisor on $X$. Let $j: U=X \backslash C \subset X$ be the open immersion. We use the same notation as in the last part of the previous section. Take an effective Cartier divisor

$$
D=\sum_{\lambda \in I} m_{\lambda} C_{\lambda} \quad \text { with } m_{\lambda} \geq 0 .
$$

Let $I^{\prime}=\left\{\lambda \in I|p| m_{\lambda}\right\}$, and put

$$
D^{\prime}=\sum_{\lambda \in I^{\prime}}\left(m_{\lambda}+1\right) C_{\lambda}+\sum_{\lambda \in I \backslash I^{\prime}} m_{\lambda} C_{\lambda} .
$$


Let $Y$ be a smooth hypersurface section such that $Y \times{ }_{X} C$ is a reduced simple normal crossing divisor on $Y$.

Definition 3.1. (1) Assuming $\operatorname{dim}(X) \geq 3$, we say that $Y$ is sufficiently ample for $(X, D)$ if the following conditions hold:

(A1) $H^{i}\left(X, \Omega_{X}^{d}(-\Xi+Y)\right)=0$ for any effective Cartier divisor $\Xi \leq D$ and for $i=d, d-1, d-2$.

(A2) For any $\lambda \in I^{\prime}$, we have

$$
\begin{aligned}
H^{0}\left(C_{\lambda}, \Omega_{X}^{1}\left(D^{\prime}-Y\right) \otimes 0_{C_{\lambda}}\right) & =H^{0}\left(C_{\lambda}, O_{C_{\lambda}}\left(D^{\prime}-Y\right)\right) \\
& =H^{1}\left(C_{\lambda}, O_{C_{\lambda}}\left(D^{\prime}-2 Y\right)\right)=0 .
\end{aligned}
$$

(2) Assuming $\operatorname{dim}(X)=2$, we say that $Y$ is sufficiently ample for $(X, D)$ if the following condition holds:

(B) $H^{i}\left(X, \Omega_{X}^{d}(-\Xi+Y)\right)=0$ for any effective Cartier divisor $\Xi \leq D$ and for $i=1,2$.

We remark that there is an integer $N$ such that any smooth $Y$ of degree $\geq N$ is sufficiently ample for $(X, D)$.

Theorem 1.1 is a direct consequence of the following:

Theorem 3.2. Let $Y$ be sufficiently ample for $(X, D)$. Write $E=Y \times_{X} D$.

(1) Assuming $d:=\operatorname{dim}(X) \geq 3$, we have isomorphisms

fil $_{D} H^{1}(U) \stackrel{\sim}{\rightarrow} \operatorname{fil}_{E} H^{1}(U \cap Y) \quad$ and $\quad \operatorname{fil}_{D}^{\log } H^{1}(U) \stackrel{\sim}{\rightarrow} \operatorname{fil}_{E}^{\log } H^{1}(U \cap Y)$.

(2) Assuming $d=2$, we have injections

$\mathrm{fil}_{D} H^{1}(U) \hookrightarrow \mathrm{fil}_{E} H^{1}(U \cap Y) \quad$ and $\quad \mathrm{fil}_{D}^{\log } H^{1}(U) \hookrightarrow \mathrm{fil}_{E}^{\log } H^{1}(U \cap Y)$.

For an abelian group $M$, we let $M\left\{p^{\prime}\right\}$ denote the prime-to- $p$ torsion part of $M$.

Lemma 3.3. (1) Assuming $d:=\operatorname{dim}(X) \geq 3$, we have an isomorphism

$$
\operatorname{fil}_{D} H^{1}(U)\left\{p^{\prime}\right\} \stackrel{\sim}{\rightarrow} \operatorname{fil}_{E} H^{1}(U \cap Y)\left\{p^{\prime}\right\}
$$

and the same isomorphism for $\mathrm{fil}_{D}^{\log }$.

(2) Assuming $d=2$, we have an injection

$$
\mathrm{fil}_{D} H^{1}(U)\left\{p^{\prime}\right\} \hookrightarrow \mathrm{fil}_{E} H^{1}(U \cap Y)\left\{p^{\prime}\right\}
$$

and the same injection for fil $_{D}^{\log }$.

Proof. Noting

$$
\text { fil }_{D} H^{1}(U)\left\{p^{\prime}\right\}=\operatorname{fil}_{C} H^{1}(U)\left\{p^{\prime}\right\}=\operatorname{fil}_{C}^{\log } H^{1}(U)\left\{p^{\prime}\right\}=\operatorname{fil}_{D}^{\log } H^{1}(U)\left\{p^{\prime}\right\},
$$

this follows from the tame case of Theorem 1.1 due to [Schmidt and Spieß 2000]. 
By the above lemma, Theorem 3.2 is reduced to the following:

Theorem 3.4. Let the assumption be as in Theorem 3.2. Take an integer $n>0$.

(1) Assuming $d:=\operatorname{dim}(X) \geq 3$, we have isomorphisms

$$
\mathrm{fil}_{D} H^{1}(U)\left[p^{n}\right] \stackrel{\sim}{\rightarrow} \operatorname{fil}_{E} H^{1}(U \cap Y)\left[p^{n}\right]
$$

and the same isomorphism for fil ${ }_{D}^{\log }$.

(2) Assuming $d=2$, we have an injection

$$
\mathrm{fil}_{D} H^{1}(U)\left[p^{n}\right] \hookrightarrow \mathrm{fil}_{E} H^{1}(U \cap Y)\left[p^{n}\right]
$$

and the same injection for $\mathrm{fil}_{D}^{\log }$.

In what follows, we consider an effective Cartier divisor with $\mathbb{Z}[1 / p]$-coefficient:

$$
D=\sum_{\lambda \in I} m_{\lambda} C_{\lambda}, \quad m_{\lambda} \in \mathbb{Z}[1 / p]_{\geq 0} .
$$

We put

$$
[D]=\sum_{\lambda \in I}\left[m_{\lambda}\right] C_{\lambda} \quad \text { with }\left[m_{\lambda}\right]=\max \left\{i \in \mathbb{Z} \mid i \leq m_{\lambda}\right\}
$$

and $\mathscr{F}( \pm D)=\mathscr{F} \otimes_{\mathcal{O}_{X}} \mathcal{O}_{X}( \pm[D])$ for an $\mathcal{O}_{X}$-module. For $D$ as above, let fil ${ }_{D}^{\log } W_{n} \mathcal{O}_{X}$ be the subsheaf of $j_{*} W_{n} \mathcal{O}_{U}$ of local sections

$$
\underline{a} \in W_{n} \hat{O}_{U} \quad \text { such that } \underline{a} \in \mathrm{fil}_{m_{\lambda}}^{\log } W_{n}\left(K_{\lambda}\right) \text { for any } \lambda \in I,
$$

where fil $m_{\lambda} W_{n}\left(K_{\lambda}\right):=\mathrm{fil}_{\left[m_{\lambda}\right]}^{\log } W_{n}\left(K_{\lambda}\right)$ is defined in Section 2 for the henselization $K_{\lambda}$ of $K=k(X)$ at $\lambda$. We note

$$
\mathrm{O}_{X}(D)=\mathrm{fil}_{D}^{\log } W_{n} \mathrm{O}_{X} \quad \text { for } n=1 .
$$

The following facts are easily checked:

- The Frobenius $F$ induces $F: \mathrm{fil}_{D / p}^{\log } W_{n} \mathrm{O}_{X} \rightarrow \mathrm{fil}_{D}^{\log } W_{n} \mathrm{O}_{X}$.

- The Verschiebung $V$ induces $V: \operatorname{fil}_{D}^{\log } W_{n-1} \mathcal{O}_{X} \rightarrow \mathrm{fil}_{D}^{\log } W_{n} \mathrm{O}_{X}$.

- The restriction $R$ induces $R: \mathrm{fil}_{D}^{\log } W_{n} \mathrm{O}_{X} \rightarrow \mathrm{fil}_{D / p}^{\log } W_{n-1} \mathfrak{O}_{X}$.

- The following sequence is exact:

$$
0 \rightarrow \mathrm{O}_{X}(D) \stackrel{V^{n-1}}{\longrightarrow} \mathrm{fil}_{D}^{\log } W_{n} \mathrm{O}_{X} \stackrel{R}{\rightarrow} \mathrm{fil}_{D / p}^{\log } W_{n-1} \mathrm{O}_{X} \rightarrow 0 .
$$

We define an object $\left(\mathbb{Z} / p^{n} \mathbb{Z}\right)_{X \mid D}$ of the derived category $D^{b}(X)$ of bounded complexes of étale sheaves on $X$ :

$$
\left(\mathbb{Z} / p^{n} \mathbb{Z}\right)_{X \mid D}=\mathrm{Cone}\left(\mathrm{fil}_{D / p}^{\log } W_{n} \mathcal{O}_{X} \stackrel{1-F}{\longrightarrow} \mathrm{fil}_{D}^{\log } W_{n} \mathcal{O}_{X}\right)[-1] .
$$


We have a distinguished triangle in $D^{b}(X)$ :

$$
\left(\mathbb{Z} / p^{n} \mathbb{Z}\right)_{X \mid D} \rightarrow \mathrm{fil}_{D / p}^{\log } W_{n} \mathcal{O}_{X} \stackrel{1-F}{\longrightarrow} \mathrm{fil}_{D}^{\log } W_{n} \mathcal{O}_{X} \stackrel{+}{\rightarrow} .
$$

Lemma 3.5. There is a distinguished triangle

$$
(\mathbb{Z} / p \mathbb{Z})_{X \mid D} \rightarrow\left(\mathbb{Z} / p^{n} \mathbb{Z}\right)_{X \mid D} \rightarrow\left(\mathbb{Z} / p^{n-1} \mathbb{Z}\right)_{X \mid D / p} \stackrel{+}{\rightarrow} .
$$

Proof. The lemma follows from the commutative diagram

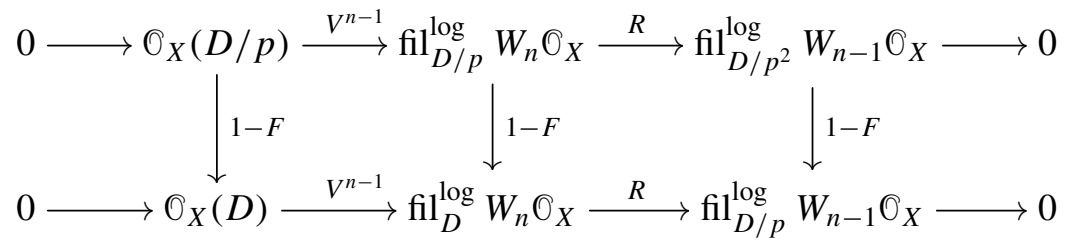

Lemma 3.6. There is a canonical isomorphism

$$
\mathrm{fil}_{D}^{\log } H^{1}(U)\left[p^{n}\right] \simeq H^{1}\left(X,\left(\mathbb{Z} / p^{n} \mathbb{Z}\right)_{X \mid D}\right) .
$$

Proof. Noting that the restriction of $\left(\mathbb{Z} / p^{n} \mathbb{Z}\right)_{X \mid D}$ to $U$ is $\mathbb{Z} / p^{n} \mathbb{Z}$ on $U$, we have the localization exact sequence

$$
H^{1}\left(X,\left(\mathbb{Z} / p^{n} \mathbb{Z}\right)_{X \mid D}\right) \rightarrow H^{1}\left(U, \mathbb{Z} / p^{n} \mathbb{Z}\right) \rightarrow H_{C}^{2}\left(X,\left(\mathbb{Z} / p^{n} \mathbb{Z}\right)_{X \mid D}\right) .
$$

For the generic point $\lambda$ of $C_{\lambda}$, (3-2) gives us an exact sequence

$$
\begin{aligned}
H_{\lambda}^{1}\left(X, \mathrm{fil}_{D / p}^{\log } W_{n} \mathcal{O}_{X}\right) \stackrel{1-F}{\longrightarrow} H_{\lambda}^{1}\left(X, \operatorname{fil}_{D}^{\log } W_{n} \mathcal{O}_{X}\right) \\
\rightarrow H_{\lambda}^{2}\left(X,\left(\mathbb{Z} / p^{n} \mathbb{Z}\right)_{X \mid D}\right) \rightarrow H_{\lambda}^{2}\left(X, \mathrm{fil}_{D / p}^{\log } W_{n} \mathcal{O}_{X}\right) .
\end{aligned}
$$

By [Grothendieck 1967, Corollary 3.10] and (3-1), we have

$$
H_{\lambda}^{i}\left(X, \mathrm{fil}_{D / p}^{\log } W_{n} \mathcal{O}_{X}\right)=H_{\lambda}^{i}\left(X, \mathrm{fil}_{D}^{\log } W_{n} \mathcal{O}_{X}\right)=0 \quad \text { for } i \geq 2
$$

and

$$
\begin{aligned}
H_{\lambda}^{1}\left(X, \mathrm{fil}_{D / p}^{\log } W_{n} \mathcal{O}_{X}\right) & \simeq W_{n}\left(K_{\lambda}\right) / \mathrm{fil}_{m_{\lambda} / p}^{\log } W_{n}\left(K_{\lambda}\right), \\
H_{\lambda}^{1}\left(X, \mathrm{fil}_{D}^{\log } W_{n} \mathcal{O}_{X}\right) & \simeq W_{n}\left(K_{\lambda}\right) / \mathrm{fil}_{m_{\lambda}}^{\log } W_{n}\left(K_{\lambda}\right) .
\end{aligned}
$$

Thus, we get

$$
H_{\lambda}^{2}\left(X,\left(\mathbb{Z} / p^{n} \mathbb{Z}\right)_{X \mid D}\right) \simeq H^{1}\left(K_{\lambda}\right)\left[p^{n}\right] / \operatorname{fil}_{m_{\lambda}}^{\log } H^{1}\left(K_{\lambda}\right)\left[p^{n}\right] .
$$

Hence, Lemma 3.6 follows from (3-3) and the injectivity of

$$
H_{C}^{2}\left(X,\left(\mathbb{Z} / p^{n} \mathbb{Z}\right)_{X \mid D}\right) \rightarrow \bigoplus_{\lambda \in I} H_{\lambda}^{2}\left(X,\left(\mathbb{Z} / p^{n} \mathbb{Z}\right)_{X \mid D}\right) .
$$

This injectivity is a consequence of: 
Claim 3.7. For $x \in C$ with $\operatorname{dim}\left(0_{X, x}\right) \geq 2$, we have

$$
H_{x}^{2}\left(X,\left(\mathbb{Z} / p^{n} \mathbb{Z}\right)_{X \mid D}\right)=0 .
$$

By Lemma 3.5, it suffices to show Claim 3.7 in case $n=1$. Triangle (3-2) gives us an exact sequence

$$
H_{x}^{1}\left(X, \mathrm{O}_{X}(D)\right) \rightarrow H_{x}^{2}\left(X,(\mathbb{Z} / p \mathbb{Z})_{X \mid D}\right) \rightarrow H_{x}^{2}\left(X, \mathrm{O}_{X}(D / p)\right) \stackrel{1-F}{\longrightarrow} H_{x}^{2}\left(X, \mathrm{O}_{X}(D)\right) .
$$

If $\operatorname{dim}\left(\mathcal{O}_{X, x}\right)>2, H_{x}^{1}\left(X, \mathcal{O}_{X}(D)\right)=0$ and $H_{x}^{2}\left(X, \mathscr{O}_{X}(D / p)\right)=0$ by [Grothendieck 1967, Corollary 3.10], which implies $H_{x}^{2}\left(X,(\mathbb{Z} / p \mathbb{Z})_{X \mid D}\right)=0$ as desired.

We now assume $\operatorname{dim}\left(0_{X, x}\right)=2$. Let $(\mathbb{Z} / p \mathbb{Z})_{X}$ denote the constant sheaf $\mathbb{Z} / p \mathbb{Z}$ on $X$, and put

$$
\mathscr{F}_{X \mid D}=\operatorname{Coker}\left(\mathcal{O}_{X}(D / p) \stackrel{1-F}{\longrightarrow} \mathrm{O}_{X}(D)\right) .
$$

Note that $\mathscr{F}_{X \mid D}=0$ for $D=0$. By definition, we have a distinguished triangle

$$
(\mathbb{Z} / p \mathbb{Z})_{X} \rightarrow(\mathbb{Z} / p \mathbb{Z})_{X \mid D} \rightarrow \mathscr{F}_{X \mid D} \stackrel{+}{\rightarrow} \text {. }
$$

By [SGA 1 1971, Exposé X, Théorèm 3.1], we have $H_{x}^{2}\left(X,(\mathbb{Z} / p \mathbb{Z})_{X}\right)=0$. Hence, we are reduced to showing

$$
H_{x}^{2}\left(X, \mathscr{F}_{X \mid D}\right)=0
$$

Without loss of generality, we can assume that $D$ has integral coefficients. We prove (3-4) by induction on multiplicities of $D$ reducing to the case $D=0$. Fix an irreducible component $C_{\lambda}$ of $C$ with the multiplicity $m_{\lambda} \geq 1$ in $D$, and put $D^{\prime}=D-C_{\lambda}$. We have a commutative diagram with exact rows and columns

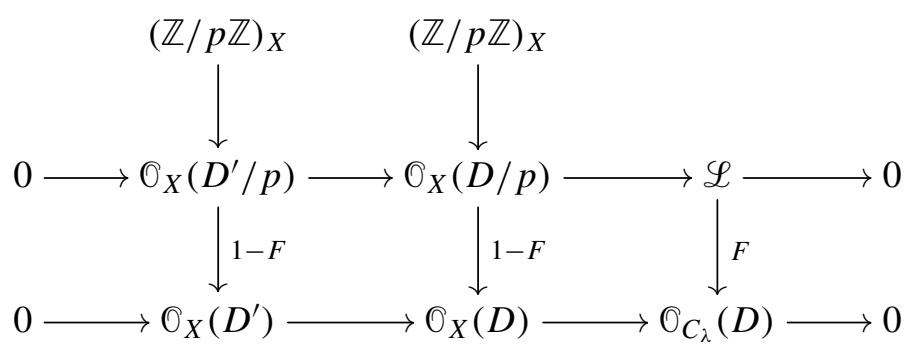

Here $\mathscr{O}_{C_{\lambda}}(D)=\mathscr{O}_{X}(D) \otimes \mathscr{O}_{C_{\lambda}}$, and $\mathscr{L}=\mathscr{O}_{C_{\lambda}}(D / p)$ if $p \mid m_{\lambda}$, and $\mathscr{L}=0$ otherwise. Thus, we get short exact sequences

$$
\begin{array}{ll}
0 \rightarrow \mathscr{F}_{X \mid D^{\prime}} \rightarrow \mathscr{F}_{X \mid D} \rightarrow \mathscr{O}_{C_{\lambda}}(D) \rightarrow 0 & \text { if } p \nmid m_{\lambda}, \\
0 \rightarrow \mathscr{F}_{X \mid D^{\prime}} \rightarrow \mathscr{F}_{X \mid D} \rightarrow \mathscr{O}_{C_{\lambda}}(D) / \mathscr{O}_{C_{\lambda}}(D / p)^{p} \rightarrow 0 & \text { if } p \mid m_{\lambda} .
\end{array}
$$


We may assume $H_{x}^{2}\left(X, \mathscr{F}_{X \mid D^{\prime}}\right)=0$ by the induction hypothesis. Hence, (3-4) follows from

$$
\begin{aligned}
H_{x}^{2}\left(C_{\lambda}, \mathscr{O}_{C_{\lambda}}(D)\right) & =0, \\
H_{x}^{2}\left(C_{\lambda}, \mathscr{O}_{C_{\lambda}}(D) / \mathscr{O}_{C_{\lambda}}(E)^{p}\right) & =0,
\end{aligned}
$$

where we put $E=[D / p]$. We may assume $x \in C_{\lambda}$ so that $\operatorname{dim}\left(0_{C_{\lambda}, x}\right)=1$ by the assumption $\operatorname{dim}\left(0_{X, x}\right)=2$. Equation (3-5) is a consequence of [Grothendieck 1967, Corollary 3.10]. In view of an exact sequence

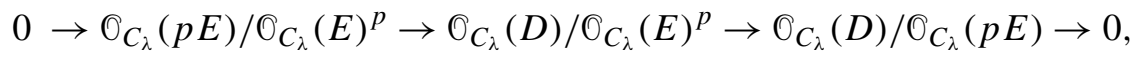

(3-6) follows from

$$
H_{x}^{2}\left(C_{\lambda}, \mathscr{O}_{C_{\lambda}}(p E) / \mathscr{O}_{C_{\lambda}}(E)^{p}\right)=0 \quad \text { and } \quad H_{x}^{2}\left(C_{\lambda}, \mathscr{O}_{C_{\lambda}}(D) / \mathscr{O}_{C_{\lambda}}(p E)\right)=0 .
$$

The first assertion follows from [Grothendieck 1967, Corollary 3.10] noting that ${ }^{O_{C}} C_{\lambda}(p E) / O_{C_{\lambda}}(E)^{p}$ is a locally free $\mathscr{O}_{C_{\lambda}}^{p}$-module. The second assertion holds since ${ }^{O} C_{\lambda}(D) / O_{C_{\lambda}}(p E)$ is supported in a proper closed subscheme $T$ of $C_{\lambda}$ and $x$ is a generic point of $T$ if $x \in T$. This completes the proof of Lemma 3.6.

Proof of Theorem 3.4. In view of the above results, the assertions for $\mathrm{fil}_{D}^{\log }$ of Theorem 3.4(1) and (2) follow from the following:

Theorem 3.8. Let the assumption be as in Theorem 3.2. The natural map

$$
H^{1}\left(X,\left(\mathbb{Z} / p^{n} \mathbb{Z}\right)_{X \mid D}\right) \rightarrow H^{1}\left(Y,\left(\mathbb{Z} / p^{n} \mathbb{Z}\right)_{Y \mid D}\right)
$$

is an isomorphism for $d:=\operatorname{dim}(X) \geq 3$, and it is injective for $d=2$.

Proof. By Lemma 3.5, we have a commutative diagram:

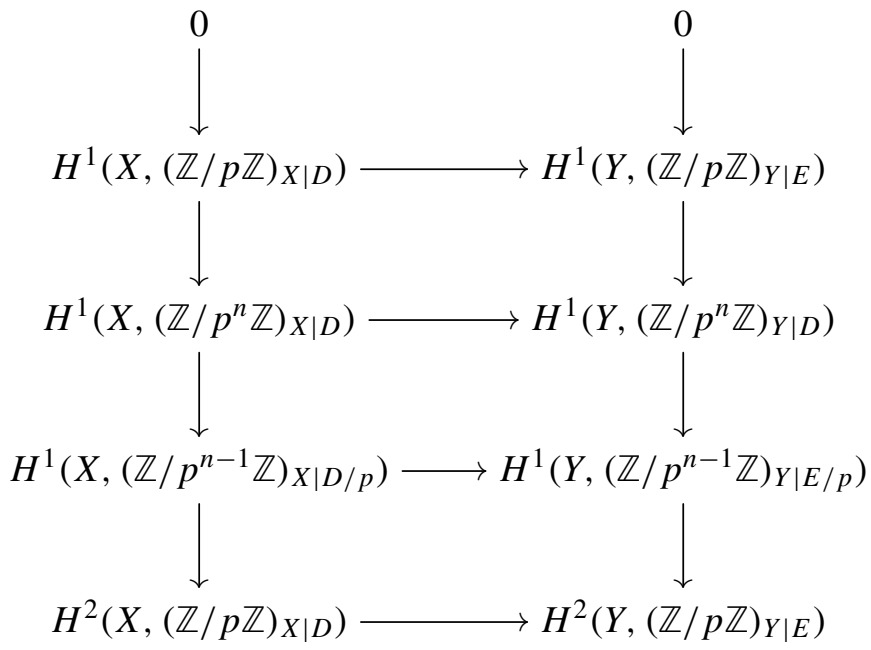


The theorem follows by the induction on $n$ from the following lemma.

Lemma 3.9. Let the assumption be as in Theorem 3.2.

(1) Assuming $d \geq 3$, the natural map

$$
H^{i}\left(X,(\mathbb{Z} / p \mathbb{Z})_{X \mid D}\right) \rightarrow H^{i}\left(Y,(\mathbb{Z} / p \mathbb{Z})_{Y \mid E}\right)
$$

is an isomorphism for $i=1$ and injective for $i=2$.

(2) Assuming $d=2$, the natural map

$$
H^{1}\left(X,(\mathbb{Z} / p \mathbb{Z})_{X \mid D}\right) \rightarrow H^{1}\left(Y,(\mathbb{Z} / p \mathbb{Z})_{Y \mid E}\right)
$$

is injective.

Proof. We define an object $\mathscr{K}$ of $D^{b}(X)$ :

$$
\mathscr{K}=\operatorname{Cone}\left(\mathrm{O}_{X}(D / p-Y) \stackrel{1-F}{\longrightarrow} \mathcal{O}_{X}(D-Y)\right)[-1] .
$$

By the commutative diagram with exact horizontal sequences

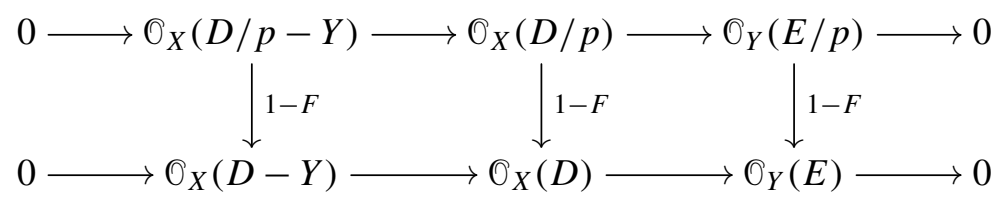

we have a distinguished triangle in $D^{b}(X)$ :

$$
\mathscr{K} \rightarrow(\mathbb{Z} / p \mathbb{Z})_{X \mid D} \rightarrow(\mathbb{Z} / p \mathbb{Z})_{Y \mid E} \stackrel{+}{\rightarrow} .
$$

Hence, it suffices to show $H^{i}(X, \mathscr{K})=0$ for $i=1,2$ in case $d \geq 3$ and $H^{1}(X, \mathscr{K})=0$ in case $d=2$. We have an exact sequence

$$
\begin{aligned}
H^{0}\left(\mathcal{O}_{X}(D-Y)\right) & \rightarrow H^{1}(X, \mathscr{K}) \rightarrow H^{1}\left(\mathcal{O}_{X}(D / p-Y)\right) \\
& \rightarrow H^{1}\left(\mathcal{O}_{X}(D-Y)\right) \rightarrow H^{2}(X, \mathscr{K}) \rightarrow H^{2}\left(\mathcal{O}_{X}(D / p-Y)\right) .
\end{aligned}
$$

By Serre duality, for a divisor $\Xi$ on $X$, we have

$$
H^{i}\left(X, \mathrm{O}_{X}(\Xi-Y)\right)=H^{d-i}\left(X, \Omega_{X}^{d}(-\Xi+Y)\right)^{\vee} .
$$

Thus, the desired assertion follows from Definition 3.1(A1) and (B).

It remains to deduce the assertions for fil $_{D}$ of Theorem 3.4(1) and (2) from those for fil ${ }_{D}^{\log }$. Let $D^{\prime}$ be as in the beginning of this section and $E^{\prime}=D^{\prime} \times_{X} Y$. Noting that the multiplicities of $D^{\prime}$ are prime to $p$, we have by Lemma 2.8(3) fil $_{D^{\prime}} H^{1}(U)=\operatorname{fil}_{D^{\prime}-C}^{\log } H^{1}(U) \quad$ and $\quad \operatorname{fil}_{E^{\prime}} H^{1}(U \cap Y)=\mathrm{fil}_{E^{\prime}-C \cap Y}^{\log } H^{1}(U \cap Y)$. 
Thus, the assertions for fil ${ }_{D^{\prime}-C}^{\text {log }}$ of Theorem 3.4 imply that for fil $D_{D^{\prime}}$. Since fil $_{D} \subset \mathrm{fil}_{D^{\prime}}$, it immediately implies the injectivity of

$$
\mathrm{fil}_{D} H^{1}(U) \rightarrow \operatorname{fil}_{E} H^{1}(U \cap Y) .
$$

It remains to deduce its surjectivity from that of

$$
\mathrm{fil}_{D^{\prime}} H^{1}(U) \rightarrow \mathrm{fil}_{E^{\prime}} H^{1}(U \cap Y)
$$

assuming $d \geq 3$. For this it, suffices to show the injectivity of

$$
\mathrm{fil}_{D^{\prime}} H^{1}(U) / \mathrm{fil}_{D} H^{1}(U) \rightarrow \mathrm{fil}_{E^{\prime}} H^{1}(U \cap Y) / \mathrm{fil}_{E} H^{1}(U \cap Y) .
$$

By Proposition 2.6, we have a commutative diagram

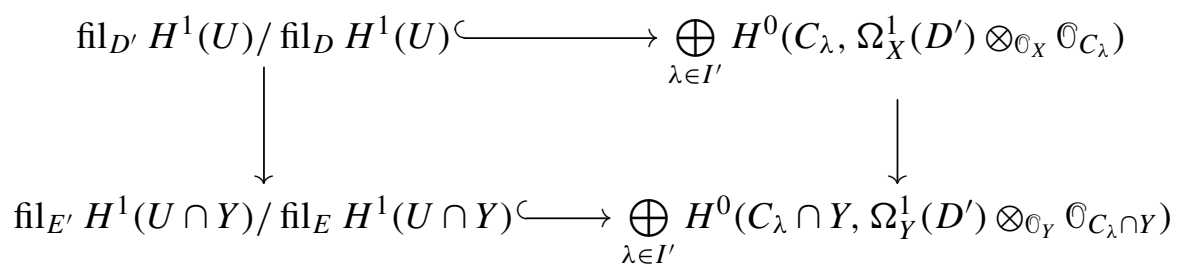

Thus, we are reduced to showing the injectivity of the right vertical map. Putting $\mathscr{L}=\operatorname{Ker}\left(\Omega_{X}^{1} \rightarrow i_{*} \Omega_{Y}^{1}\right)$ where $i: Y \subset X$, the assertion follows from

$$
H^{0}\left(C_{\lambda}, \mathscr{L}\left(D^{\prime}\right) \otimes_{0_{X}} 0_{C_{\lambda}}\right)=0 .
$$

Note that we used the fact that $Y$ and $C_{\lambda}$ intersect transversally. We have an exact sequence

$$
0 \rightarrow \Omega_{X}^{1}(-Y) \rightarrow \mathscr{L} \rightarrow \mathrm{O}_{X}(-Y) \otimes \mathrm{O}_{Y} \rightarrow 0 .
$$

From this, we get an exact sequence

$$
0 \rightarrow \Omega_{X}^{1}\left(D^{\prime}-Y\right) \otimes_{0_{X}} \mathscr{O}_{C_{\lambda}} \rightarrow \mathscr{L}\left(D^{\prime}\right) \otimes_{\mathbb{O}_{X}} \mathscr{O}_{C_{\lambda}} \rightarrow \mathscr{O}_{C_{\lambda}}\left(D^{\prime}-Y\right) \otimes \mathcal{O}_{C_{\lambda} \cap Y} \rightarrow 0 .
$$

We also have an exact sequence

$$
0 \rightarrow{ }^{\circ} C_{\lambda}\left(D^{\prime}-2 Y\right) \rightarrow{ }^{{ }^{O}} C_{\lambda}\left(D^{\prime}-Y\right) \rightarrow{ }^{{ }^{O}} C_{\lambda}\left(D^{\prime}-Y\right) \otimes{ }^{O} C_{\lambda} \cap Y \rightarrow 0 .
$$

Therefore, the desired assertion follows from Definition 3.1(A2). This completes the proof of Theorem 3.4.

\section{References}

[Esnault and Kerz 2012] H. Esnault and M. Kerz, "A finiteness theorem for Galois representations of function fields over finite fields (after Deligne)", Acta Math. Vietnam. 37:4 (2012), 531-562. MR 3058662 Zbl 06133191

[Grothendieck 1967] A. Grothendieck, Local cohomology, Lecture Notes in Mathematics 41, Springer, Berlin, 1967. MR 37 \#219 Zbl 0185.49202 
[de Jong 1996] A. J. de Jong, "Smoothness, semi-stability and alterations", Inst. Hautes Études Sci. Publ. Math. 83 (1996), 51-93. MR 98e:14011 Zbl 0916.14005

[Kato 1989] K. Kato, "Swan conductors for characters of degree one in the imperfect residue field case", pp. 101-131 in Algebraic K-theory and algebraic number theory (Honolulu, 1987), edited by M. R. Stein and R. K. Dennis, Contemp. Math. 83, Amer. Math. Soc., Providence, RI, 1989. MR 90g:11164 Zbl 0716.12006

[Kerz and Saito 2013] M. Kerz and S. Saito, "Chow group of 0-cycles with modulus and higher dimensional class field theory”, preprint, 2013. arXiv 1304.4400

[Laumon 1981] G. Laumon, "Semi-continuité du conducteur de Swan (d'après P. Deligne)", pp. 173-219 in The Euler-Poincaré characteristic, edited by J.-L. Verdier, Astérisque 83, Soc. Math. France, Paris, 1981. MR 83g:14007 Zbl 0504.14013

[Matsuda 1997] S. Matsuda, "On the Swan conductor in positive characteristic", Amer. J. Math. 119:4 (1997), 705-739. MR 99h:14022 Zbl 0928.14017

[Raskind 1995] W. Raskind, "Abelian class field theory of arithmetic schemes", pp. 85-187 in $K$ theory and algebraic geometry: connections with quadratic forms and division algebras (Santa Barbara, 1992), part 1, edited by B. Jacob and A. Rosenberg, Proc. Sympos. Pure Math. 58, Amer. Math. Soc., Providence, RI, 1995. MR 96b:11089 Zbl 0832.19004

[Schmidt and Spieß 2000] A. Schmidt and M. Spieß, "Singular homology and class field theory of varieties over finite fields", J. Reine Angew. Math. 527 (2000), 13-36. MR 2001m:11108 Zbl 0961.14013

[SGA 1 1971] A. Grothendieck, Séminaire de Géométrie Algébrique du Bois Marie 1960-1961: revêtements étales et groupe fondamental, Lecture Notes in Mathematics 224, Springer, Berlin, 1971. MR 50 \#7129 Zbl 0234.14002

[SGA 2 1968] A. Grothendieck, Séminaire de Géométrie Algébrique du Bois-Marie 1962: cohomologie locale des faisceaux cohérents et théorèmes de Lefschetz locaux et globaux, Advanced Studies in Pure Mathematics 2, North-Holland, Amsterdam, 1968. MR 57 \#16294 Zbl 0197.47202

Communicated by Jean-Louis Colliot-Thélène

Received 2013-04-27 Revised 2013-11-12 Accepted 2013-12-10

moritz.kerz@mathematik.uni-regensburg.de

Naturwissenschaftliche Fakultät I - Mathematik, Universität Regensburg, 93040 Regensburg, Germany

sshuji@msb.biglobe.ne.jp Interactive Research Center of Science, Graduate School of Science and Engineering, Tokyo Institute of Technology, Ookayama, Meguro, Tokyo 152-8551, Japan 


\section{Algebra \& Number Theory}

msp.org/ant

\section{EDITORS}

MANAGING EDITOR

Bjorn Poonen

Massachusetts Institute of Technology

Cambridge, USA

\author{
EDITORIAL BOARD CHAIR \\ David Eisenbud \\ University of California \\ Berkeley, USA
}

\section{BOARD OF EDITORS}

Georgia Benkart

Dave Benson

Richard E. Borcherds

John H. Coates

J-L. Colliot-Thélène

Brian D. Conrad

Hélène Esnault

Hubert Flenner

Edward Frenkel

Andrew Granville

Joseph Gubeladze

Roger Heath-Brown

Ehud Hrushovski

Craig Huneke

Mikhail Kapranov

Yujiro Kawamata

János Kollár

Yuri Manin

Barry Mazur

Philippe Michel
University of Wisconsin, Madison, USA

University of Aberdeen, Scotland

University of California, Berkeley, USA

University of Cambridge, UK

CNRS, Université Paris-Sud, France

University of Michigan, USA

Freie Universität Berlin, Germany

Ruhr-Universität, Germany

University of California, Berkeley, USA

Université de Montréal, Canada

San Francisco State University, USA

Oxford University, UK

Hebrew University, Israel

University of Virginia, USA

Yale University, USA

University of Tokyo, Japan

Princeton University, USA

Northwestern University, USA

Harvard University, USA

École Polytechnique Fédérale de Lausanne
Susan Montgomery

Shigefumi Mori

Raman Parimala

Jonathan Pila

Victor Reiner

Karl Rubin

Peter Sarnak

Joseph H. Silverman

Michael Singer

Vasudevan Srinivas

J. Toby Stafford

Bernd Sturmfels

Richard Taylor

Ravi Vakil

Michel van den Bergh

Marie-France Vignéras

Kei-Ichi Watanabe

Efim Zelmanov

Shou-Wu Zhang
University of Southern California, USA

RIMS, Kyoto University, Japan

Emory University, USA

University of Oxford, UK

University of Minnesota, USA

University of California, Irvine, USA

Princeton University, USA

Brown University, USA

North Carolina State University, USA

Tata Inst. of Fund. Research, India

University of Michigan, USA

University of California, Berkeley, USA

Harvard University, USA

Stanford University, USA

Hasselt University, Belgium

Université Paris VII, France

Nihon University, Japan

University of California, San Diego, USA

Princeton University, USA

PRODUCTION

production@msp.org

Silvio Levy, Scientific Editor

See inside back cover or msp.org/ant for submission instructions.

The subscription price for 2014 is US $\$ 225 /$ year for the electronic version, and $\$ 400 /$ year $(+\$ 55$, if shipping outside the US) for print and electronic. Subscriptions, requests for back issues and changes of subscribers address should be sent to MSP.

Algebra \& Number Theory (ISSN 1944-7833 electronic, 1937-0652 printed) at Mathematical Sciences Publishers, 798 Evans Hall \#3840, c/o University of California, Berkeley, CA 94720-3840 is published continuously online. Periodical rate postage paid at Berkeley, CA 94704, and additional mailing offices.

ANT peer review and production are managed by EditFLOW ${ }^{\circledR}$ from Mathematical Sciences Publishers.

\section{PUBLISHED BY}

- mathematical sciences publishers

nonprofit scientific publishing

http://msp.org/

(C) 2014 Mathematical Sciences Publishers 


\section{Algebra \& Number Theory}

Volume $8 \quad$ No. $3 \quad 2014$

Derived invariants of irregular varieties and Hochschild homology

LUIGI LOMBARDI

Sato-Tate distributions of twists of $y^{2}=x^{5}-x$ and $y^{2}=x^{6}+1$

FRANCESC Fité and ANDREW V. SUTHERLAND

The algebraic dynamics of generic endomorphisms of $\mathbb{P}^{n}$

NAJMUDDIN FAKHRUDDIN

The tame-wild principle for discriminant relations for number fields

JOHN W. JONES and DAVID P. ROBERTS

Linear forms in logarithms and integral points on higher-dimensional varieties

AARON LEVIN

Lefschetz theorem for abelian fundamental group with modulus

689

MORITZ KERZ and ShuJi SAITO

Localization of spherical varieties

703

FRIEDRICH KNOP

Lefschetz operator and local Langlands modulo $\ell$ : the limit case

JEAN-FRANÇOIS DAT

Splitting tower and degree of tt-rings

PAUL BALMER 